IdeAs

Idées d'Amériques

8 | 2016

Ressources minières dans les Amériques: Mutations

d'un continent

\title{
Diego Rivera : Autoportrait de l'artiste en révolutionnaire ou en créateur
}

\section{Ana Cecilia Hornedo Marín}

\section{(2) OpenEdition}

\section{Journals}

Édition électronique

URL : https://journals.openedition.org/ideas/1732

DOI : $10.4000 /$ ideas. 1732

ISSN : 1950-5701

Éditeur

Institut des Amériques

\section{Référence électronique}

Ana Cecilia Hornedo Marín, « Diego Rivera : Autoportrait de l'artiste en révolutionnaire ou en créateur », IdeAs [En ligne], 8 | 2016, mis en ligne le 20 décembre 2016, consulté le 18 octobre 2022. URL : http:// journals.openedition.org/ideas/1732 ; DOI : https://doi.org/10.4000/ideas.1732

Ce document a été généré automatiquement le 18 octobre 2022.

\section{(c)}

Creative Commons - Attribution - Pas d'Utilisation Commerciale - Pas de Modification 4.0 International - CC BY-NC-ND 4.0

https://creativecommons.org/licenses/by-nc-nd/4.0/ 


\title{
Diego Rivera : Autoportrait de l'artiste en révolutionnaire ou en créateur
}

\author{
Ana Cecilia Hornedo Marín
}

1 À l'issue du processus révolutionnaire (1910-1920), José Vasconcelos, ministre de l'Éducation de 1920 à 1924, fait appel aux artistes afin qu'ils se joignent au programme qui fournira les bases d'un nationalisme révolutionnaire. Il ne s'agissait pas seulement de l'édification d'un nouvel État mais de la formation d'un homme nouveau ou «homme régénéré »1.

2 L'une des richesses de l'exposition du Grand Palais réside dans ses portraits. Sans les passer en revue, je voudrais montrer en quoi les portraits, et surtout les autoportraits, soulèvent une série de questions sur la mise en forme et en images de l'homme nouveau révolutionnaire, laquelle s'inscrira durablement dans le nationalisme mexicain sous le signe de l'idéologie du métissage.

On peut noter comment les artistes, notamment les "trois grands", Diego Rivera, David Alfaro Siqueiros et José Clemente Orozco, se considéraient comme au carrefour d'une triple révolution : politico-sociale, artistique et culturelle. Dans cet article nous nous concentrerons sur Diego Rivera, l'artiste le plus emblématique du mouvement muraliste, afin de montrer comment l'artiste participe à la mise en forme du nationalisme mexicain et en quoi son positionnement en tant qu'artiste révolutionnaire est hautement ambigu.

Diego Rivera, à 24 ans, était reconnu en tant qu'artiste lorsque la révolution a commencé en 1910. Pendant toute la période de la révolution proprement dite, comme la plupart des artistes mexicains reconnus, il vivait en Europe, où il étudiait dans des écoles d'art moderne. Ses écrits montrent à quel point les luttes révolutionnaires du Mexique étaient à cette époque loin de ses préoccupations. Il aspirait à se faire une place dans le monde artistique européen où il était arrivé en 1907. Son œuvre de ces années en témoigne, aussi bien par sa technique que par son contenu. Une peinture fait 
exception : El guerrillero, tableau cubiste peint en 1915 alors qu'il vivait en Espagne et auquel, des années plus tard, il donna le titre de Paisaje zapatista.

5 En Europe, Diego Rivera participe à différents courants esthétiques et aux discussions métaphysiques d'un groupe d'artistes et d'émigrés russes. C'est ainsi qu'il fait la connaissance de l'écrivain Ilya Ehrenbourg, qui sera le premier auteur à adopter le peintre comme personnage d'un roman, Les Aventures extraordinaires de Julio Jurenito (Ehrenbourg I., 1964). Ce texte, écrit sous forme de farce, préfigure la manière dont Rivera construira son propre personnage en se dépeignant comme l'exemple même de l'audace, de l'irrévérence et de l'excentricité face à l'ordre bourgeois. Plus tard, Rivera emploiera fréquemment le mot « révolution » pour parler de son rapport à l'art. Mais de quelle révolution s'agit-il ? Pour répondre à cette question, nous nous appuierons d'abord sur ses écrits sur l'art, ensuite sur son autobiographie (Rivera D. et March G., 1991), puis sur la biographie écrite par Bertram D. Wolfe (1994). Enfin, nous interrogerons l'image de l'artiste telle qu'elle se donne à voir dans ses autoportraits.

\section{Les écrits de Rivera sur l'art}

Dans certains de ses articles, se référant à son œuvre et à sa vision de l'art, Diego Rivera parle de lui-même à la troisième personne, comme d'un personnage qu'il met en scène. En revanche, quand il parle de son militantisme au Parti communiste, c'est à la première personne ${ }^{2}$. Depuis ses premiers articles écrits après son retour au Mexique en 1922 jusqu'à ses derniers essais en 1957, il définit l'art comme une nécessité biologique, une forme de «nutrition» (Rivera D., 1979: 209). La révolution est définie comme l'avenir : celui qui appartient au prolétariat et devra être dirigé, puisque les masses ont besoin d'être organisées, d'où la nécessité de l'art révolutionnaire. Dans son article « La révolution en peinture» (1929) il affirme que «le problème des artistes révolutionnaires consiste à offrir une forme esthétique adéquate à l'alimentation esthétique...». Pour cela, l'artiste révolutionnaire doit utiliser sa technique ultramoderne et permettre que son éducation classique (s'il l'a eue) l'affecte subconsciemment.

C'est pourquoi il n'y a pas de raison de s'effrayer que le thème soit admis comme une nécessité primordiale, l'artiste est absolument libre de créer une œuvre d'art totalement plastique. Le thème est au peintre ce que les rails sont à la locomotive (Rivera D., 1996 : 107).

8 Ainsi, il choisit librement les thèmes qu'il traite. Indépendamment, sans contrainte exercée par le Parti communiste, mais souvent avec l'accord de celui-ci, Rivera a produit sur les murs une œuvre iconographique souvent qualifiée de " révolutionnaire ». En quoi l'était-elle? Rivera s'est chargé par exemple de récupérer l'art populaire, comme les gravures de crânes (calaveras) de José Guadalupe Posada. Il a mis en scène le prolétariat et les machines industrielles. S'il a mis en évidence par ses images le monde indigène, il s'agissait moins d'aller à la rencontre de ce monde que de prôner son intégration au monde occidental. La révolution mexicaine elle-même est mise en images à travers la trinité révolutionnaire : le paysan, l'ouvrier et le soldat. On trouve dans certaines œuvres des images d'Emiliano Zapata, mais en dehors de tout contexte de lutte.

Rivera ne valorise pas dans ses écrits la spontanéité et la radicalité des luttes sociales, notamment dans les campagnes. Pour lui la révolution est plutôt synonyme d'un programme guidé. Il conçoit son œuvre comme s'inscrivant dans une période où les forces populaires sont soumises à un nouvel État qu'il qualifie de bourgeois, dont le 
contenu correspond plus à une modernisation du capitalisme qu'à un régime socialiste et prolétarien. Il justifie sa collaboration avec ce régime en écrivant, en 1928 :

Il était clair que ce gouvernement ferait tout ce qui était nécessaire pour conserver la sympathie du prolétariat qui l'avait aidé à parvenir au pouvoir. Il était donc possible qu'il acceptât la proposition de faire des expériences de peinture murale monumentale de caractère révolutionnaire (ibid., p. 91).

Bien que, à diverses occasions, Diego Rivera définisse les artistes en général, lui-même inclus, comme des travailleurs, il se met en scène comme guide ou maître, en écrivant à maintes reprises des phrases comme celle-ci, qui date de 1928 :

Je voudrais que mes peintures reflètent la vie sociale du Mexique tel que je le voyais et qu'à travers la réalité et l'ordre de ce reflet de l'authentique, les possibilités pour le futur soient désignées aux masses. J'essayais toujours d'être ce qu'on appelle un artiste, c'est-à-dire un récepteur de la lutte et des désirs de la masse et un transmetteur qui lui offre la synthèse de ses désirs pour ainsi lui servir de conscience et l'aider dans son organisation sociale (ibid., p. 97).

11 Ainsi, pour Diego Rivera, le peintre peut interpréter les désirs des masses et en leur donnant des formes plastiques, il devient leur créateur. Il relève de la figure de l'artiste créateur au sens de Jean-Marie Schaeffer, à savoir l'artiste investi d'une mission historique ou eschatologique qui consiste à incarner par ses œuvres Dieu dans le monde. Schaeffer parle de «l'idéologie de l'artiste créateur, née de sa réinterprétation du domaine artistique dans le champ de la politique » (Heinich N. et Schaeffer J.-M., 2004 : 97).

L'autobiographie de Diego Rivera My Art, My Life, dictée par Rivera et retranscrite par Gladys March entre 1944 et 1957, présente toutes les difficultés d'interprétation liées à ce genre. Les autobiographies d'artistes, comme le signale Philippe Lejeune (1971:47), conviennent moins que la correspondance à la transposition de l'expérience picturale de l'artiste. Le manque de contexte social et politique dans My Art, My Life rend ardue une interprétation de la revendication par Rivera du statut d'artiste révolutionnaire. Le peintre parle abondamment de ses relations avec les femmes. Il revient constamment sur sa relation à son corps, son gros ventre, ses yeux de crapaud et son sourire. Il fait part de nombreuses anecdotes à propos de l'enfant prodige et anticonformiste qu'il a été, contribuant ainsi à la formation de sa propre légende, comme le diraient Ernst Kris et Otto Kurz (1979). Certains passages mélangent des propos irrévérents sur la bourgeoisie et des allusions à son militantisme. Ce sont les seuls qui justifient qu'on puisse parler de lui comme d'un artiste révolutionnaire.

Néanmoins, cette autobiographie nous permet de comprendre comment le personnage que Rivera a créé en parlant de lui-même est porteur d'une vision idéologique de l'Homme nouveau révolutionnaire. Cet homme-là est représenté comme un métis, le produit d'un père espagnol et d'une mère indigène. Rivera le souligne en effet dès la première page, en parlant de son père d'origine espagnole et en évoquant son amour pour la nourrice indigène qui s'occupait de lui comme une mère. Notons au passage comment les dimensions de race et de genre se combinent dans cette image: le masculin est associé à l'Européen tandis que l'indigène est nécessairement féminisé.

Diego Rivera interroge peu dans ce texte la réception de son œuvre par les classes populaires. Il ne renie pas non plus son activité d'autopromotion aux États-Unis auprès de collectionneurs et de commanditaires capitalistes tels que Ford et Rockefeller. En revanche, il prête beaucoup d'attention aux entrées et sorties d'argent, préoccupation 
qu'il justifie en disant que l'argent lui permet de financer de nouvelles fresques ou bien de soutenir le Parti communiste.

Rivera ne s'engage dans aucune réflexion iconologique sur son œuvre mais reste dans le registre de la description iconographique. Par exemple, il reste muet sur l'étouffement du mouvement zapatiste et plus généralement sur la soumission du mouvement révolutionnaire au parti hégémonique, le Parti révolutionnaire institutionnel. En revanche, il se pose en prophète, en soutenant que par son œuvre et par son expérience il a pu prévoir les calamités du fascisme, de la Deuxième Guerre mondiale, et préfigurer la fission nucléaire (Wolfe B. D., 1994: 90). My Art, My Life résume, en somme, la construction du personnage qui s'est emparé de Diego Rivera et l'a rendu prisonnier de ses propres fables, dès ses années à Paris. On comprendra mieux cependant l'importance politique de ces années grâce au biographe Bertram D. Wolfe.

\section{Biographie}

Bertram D. Wolfe, commentateur politique, et Élie Faure, historien de l'art, sont les deux personnes qui ont le plus influencé Rivera intellectuellement ${ }^{3}$. Wolfe, jeune militant communiste à l'époque où il a rencontré Rivera, fut l'un des premiers à valoriser l'œuvre du peintre. La biographie, publiée en 1963, à une époque où il était devenu un militant anticommuniste connu, profite de la distance critique et d'un patient travail intellectuel. Pour comprendre le parcours de Rivera et son rapport à l'idée de révolution, c'est une source de première importance. Wolfe explique ainsi son titre, La Vie fabuleuse de Diego Rivera :

19 Puisque la personne et le volume physique et corporel de Diego étaient fabuleux. Sa vie a été fabuleuse, et le récit qu'il en fait, encore plus fabuleux [...] et la manière dont il les narrait à différentes reprises, étaient un interminable labyrinthe de fables. Ses peintures sur les murs des bâtiments publics de Mexico constituent une longue et trompeuse fable sur le monde, l'époque, et le passé, présent et avenir de son pays (1994: 19).

Mais - écrit-il aussi -,

il faisait ses récits avec tant d'habileté, avec un tel air de franchise et de luxe de détails qu'il était difficile de ne pas devenir complice, de ne pas faire comme si narrateur et auditeur croyaient à ce qu'il disait (ibid., p. 20).

Comme Wolfe le signale, les influences idéologiques reçues par Diego Rivera, toujours d'origine européenne (l'anarcho-syndicalisme espagnol, le populisme russe, etc.) furent assimilées sans rigueur. Rivera aurait fait sa première découverte de Marx à travers une mauvaise traduction du Capital qu'il n'a jamais fini de lire, de même qu'il a feuilleté de nombreux autres livres sans les avoir vraiment lus et que les nuances de la pensée de Marx ou de Lénine lui importaient peu. Ainsi, écrivait Wolfe :

La révolution qui s'est emparé de Diego Rivera et a fait de lui un peintre était la Révolution mexicaine et non la russe. La Révolution mexicaine a été, avant tout, une tentative d'autodécouverte [du Mexique] [...] Tout cela signifie que Diego Rivera était davantage populiste que communiste. Sa peinture le démontre (ibid.,p. 332-333).

Ici nous retrouvons l'une des interprétations de la révolution mexicaine les plus influentes, et qui mériterait d'être interrogée à son tour : la révolution comme miroir du plus profond de l'être de la société, telle qu'Octavio Paz en avait parlé dans Le Labyrinthe de la solitude (1950). Cette interprétation a inspiré beaucoup de créations littéraires et artistiques sur la révolution et l'art, parmi lesquelles le récit 
hagiographique de J.M.G. Le Clézio intitulé Diego et Frida, où le romancier défend Rivera comme révolutionnaire en raison même de sa glorification du monde indigène. Il écrit : «À partir de 1923 [...] Rivera montre ce qu'il recherche dans la réalité indigène : le peuple opprimé porte en lui la révolution, la reconquête de sa liberté, [...] C'est dans cette réalité que Diego Rivera puise les éléments de sa foi révolutionnaire » (1993: 254). Rivera a en effet rendu visible le monde indigène en l'intégrant dans ses fresques, mais il l'a fait précisément à la manière du populisme latino-américain dans le domaine de l'art: le pouvoir est représenté comme partant du "peuple» et de ses luttes, mais celles-ci sont contrôlées par en haut, par la figure du dirigeant révolutionnaire "à l'écoute du peuple». C'est ici que l'on voit le caractère prophétique de l'œuvre artistique de Rivera.

\section{Autoportraits}

Si un terme autre que "révolutionnaire " peut résumer les écrits de Diego Rivera, c'est celui de "fabulateur" (hâbleur, invencionero ou inventeur d'histoires, mythomane), compte tenu de ses innombrables anecdotes (Hornedo A. C., 2014). Néanmoins, dans ses autoportraits peints (sur lesquels il n'a rien écrit ou déclaré, à notre connaissance), il a exprimé avec une certaine honnêteté son image en tant qu'artiste.

Rivera a réalisé plus de vingt autoportraits entre 1906 et 1951 (Moyssén X., 1989). Tantôt il signe simplement « D. R. » ou « Diego Rivera », tantôt il y a une dédicace, mais aussi, parfois, la faucille et le marteau. On ne retrouve plus le personnage fantasque de ses anecdotes, «Diego María de la Concepción Juan NepomucenoEstanislao de la Rivera y Barrientos Acosta y Rodríguez ».

Ce sont surtout ses quatre autoportraits les plus significatifs réalisés sur des fresques qui nous renseignent sur la place qu'il occupe dans un espace pictural qu'il appelait "révolutionnaire ». Il ne porte jamais sur lui le signe de la faucille et du marteau ou d'autres signes d'engagement dans la lutte. La position de classe qu'il occupe est celle d'un bourgeois, en tant qu'observateur, face au peuple unifié par la fête et la tradition, comme dans la fresque de 1923, La Fête des morts. Et, plus tard, dans les deux autoportraits réalisés dans Rêve d'un dimanche après-midi dans le parc Alameda (1948), il retourne à son enfance. Il évoque l'inspiration des calaveras de Posada. En accordant à de telles images issues de l'art populaire sa reconnaissance d'artiste, en les intégrant dans un art plus recherché, il entend faire œuvre révolutionnaire.

Évoquons, pour conclure, deux autoportraits significatifs du point de vue de l'image de Diego Rivera en tant qu'artiste. En 1926, il se représente avec un corps complet pour la première fois, en compagnie d'un sculpteur et d'un peintre. Il se dépeint en architecte, en s'inspirant d'une photo d'Edward Weston. Pour Rivera la fresque est en effet essentiellement architecturale: dans un essai de 1934 il parle de l'architecture commede « l'élément d'union et d'amalgame entre cette machine qu'est l'édifice et la société humaine qui l'utilise » (Rivera D., 1996 : 166). Enfin, en 1931 à l'Institut d'art de San Francisco, il se représente comme un " peintre de fresques » qui « se connaît si bien qu'il arrive même à peindre son derrière ", comme le dit Wolfe. Il représente ici l'action collective de toute œuvre artistique, dimension que l'on ne retrouve pas dans ses écrits, où domine son individualisme. Peut-être s'agit-il ici de la révolution artistique au sens où l'entend Howard Becker dans Les Mondes de l'art (2006: 59).Pour ce sociologue, l'artiste est au centre d'une chaîne de coopération liant tous ceux qui, à des degrés divers, concourent à l'existence de l'œuvre. 
C'est ainsi que Rivera, tout en proclamant la révolution, participe, sans en être tout à fait conscient, de la mise en place de l'ordre étatique post-révolutionnaire sous la direction d'un parti autoritaire. En mettant en scène l'action politique dans ses fresques et en invitant le spectateur à s'y identifier, il s'associe à une révolution qui est en réalité un processus post-révolutionnaire de construction étatique qui intègre les citoyens en les contrôlant. Les lignes du dessin et l'harmonie de ses portraits reflètent bien cette orientation de son œuvre.

\section{BIBLIOGRAPHIE}

Becker, Howard, Les Mondes de l'art, Paris, Flammarion, 2006 [1986].

Ehrenbourg, Ilya, Les Aventures extraordinaires de Julio Jurenito, roman traduit du russe par Denise Meunier [éd. orig. Moscou], Paris, Plon, 1964 [1923].

Fell, Claude, José Vasconcelos: Los años de águila, México, UNAM, 1989.

Kris, Ernst, et Otto Kurz, L'Image de l'artiste. Légende, mythe et magie, Paris, Rivages, 1979.

Heinich, Nathalie, et Jean-Marie Schaeffer, Art, création, fiction. Entre sociologie et philosophie, Nîmes, Jacqueline Chambon, 2004.

Hornedo, Ana Cecilia, «À quoi joue Diego Rivera? », inAnne Gimbert, Lorenzo Lorenzo-Martín (dir.), Le Jeu : Ordre et Liberté, Le Mans, Cénomane / ALMOREAL, 2014, p. 125-132.

Lejeune, Philippe, L'Autobiographie en France, Paris, Armand Colin, 1971.

Le Clézio, Jean-Marie Gustave, Diego et Frida, Paris, Gallimard, 1993.

Moyssén Xavier, “Los autorretratos de Diego Rivera”, Anales del Instituto de Investigaciones Estéticas, México, UNAM, XV, n 60, 1989, p. 117-128.

Paz, Octavio, Le Labyrinthe de la solitude [éd. orig. Mexico] suivi de Critique de la pyramide, éd. refondue et augm., trad. Jean-Clarence Lambert, Paris : Gallimard, 1972, 354 p. [1950].

Rivera, Diego, Arte y política, México, Grijalbo, 1979.

Rivera, Diego, Écrits sur l'art, Neuchâtel, Ides et Calendes, 1996.

Rivera, Diego et Gladys March, My Art, My Life, An Autobiography, New York, Dover Publications, 1991 [1960].

Tibol, Raquel, Diego Rivera: Luces y sombras. Narración documental, México, Lumen, 2007.

Vasconcelos, José, La Raza cósmica, Espasa-Calpe, 1966 [1925].

Wolfe, Bertram D., La Vie fabuleuse de Diego Rivera[éd. orig. The Fabulous Life of Diego Rivera, New York, Stein and Day Publishers], Paris, Séguier, 1994 [1963]. 


\section{NOTES}

1. José Vasconcelos élabora une vision de la «race ibéro-américaine » selon laquelle l'Amérique hispanique devait produire une synthèse culturelle porteuse de toutes les richesses de l'histoire. La "cinquième race» ou "race cosmique», représentant l'intégration ethnique et spirituelle de toutes les cultures présentes dans la région, devait aboutir selon lui à un nouvel ordre universel, grâce à la victoire de l'esthétique sur le politique (Vasconcelos J., 1966 ; Fell C., 1989).

2. Après plusieurs allers-retours entre le Parti communiste et la dissidence trotskiste, il est revenu au « bercail » du Parti communiste en 1954 (Tibol R., 2007: 147-148).

3. Xavier Moyssén, prologue à Wolfe B. D., 1994 : V-VI.

\section{AUTEUR}

\section{ANA CECILIA HORNEDO MARÍN}

Spécialiste en sciences sociales et en histoire de l'art, elle prépare une thèse sur la figure de l'artiste révolutionnaire au XXe siècle à travers l'exemple du muralisme mexicain, à l'École des hautes études en sciences sociales. Ses principales publications portent sur l'analyse de l'espace du politique dans la peinture mexicaine moderne. achornedo@estp-paris.eu 